\title{
New one-dimensional iterative approach to restoring two-dimensional circularly band-limited images
}

\author{
G. Gregori* and S. Wabnitz \\ Fondazione "Ugo Bordoni" and Istituto Superiore delle Poste e Telecomunicazioni, Viale Europa 160, Rome, \\ Italy
}

Received December 2, 1984; accepted June 20, 1985

\begin{abstract}
A new method for the restoration of two-dimensional (2-D) images obtained through a circularly band-limited system is given. Object and image are decomposed into circular harmonics, and it is observed that the imaging system acts separately on each harmonic. We show that superresolution is, in practice, attainable with a small number of one-dimensional iterations. The method presents several advantages on the conventional 2-D algorithms of the Gerchberg type. The computing effort in particular can be much reduced. Performances of our method on computer-generated images are presented.
\end{abstract}

\section{INTRODUCTION}

In this paper we present a new approach to restoring images obtained through a circularly band-limited optical system.

Superresolution of the image of a finite object produced by arbitrary low-pass nonnegative pupils in coherent and incoherent illumination has been a topic of current interest (see, for example, Refs. 1-4) since a simple iterative method was introduced by Gerchberg in $1974^{5}$ and further analyzed ${ }^{6,7}$ and generalized $^{8-13}$ by others.

Although a complete restoration of the information contained in the object spectrum is in theory possible only in the noise-free case with an infinite number of iterations, ${ }^{6}$ a significant improvement to the effective resolution of a real noisy image is achievable with a finite number of iterations if the number of degrees of freedom, or the space-bandwidth product, of the system is low. ${ }^{6}$ The techniques for superresolution known at present are subject to strong limitations in this sense. That is, the overall number of degrees of freedom, in a typical multidimensional system, is so high that an expensive computational effort is required, involving a large number of elementary operations as well as long access time in sequential access memories.

Observing that the circular symmetry of the system allows for the decomposition of the object-to-image relationship into a denumerable set of one-dimensional (1-D) operators that link circular harmonics of the same order, we will demonstrate here how a two-dimensional (2-D) restoration may be performed through a selected number of 1-D restorations, each of them corresponding to angular information of interest in the particular image.

The convergence of the method, as well as the effect of a finite number of iterations on the image restoration, will be analyzed by means of the generalized prolate spheroidal wave functions. ${ }^{14}$

The efficiency of the method will be checked by means of the restoration of some computer-generated test images. We have achieved good reconstructions by iterating only those few harmonic components that were present in the image in a significant way.
The main advantage of our 1-D approach is that, depending on the effective space-bandwidth product of each harmonic, it allows for a differentiation of the restoring effort.

\section{IMAGING THROUGH CIRCULARLY SYMMETRIC SYSTEMS}

We remember how a 2-D shift-invariant imaging system can be generally characterized by means of its point-spread function $s(x, y)$ :

$$
i(x, y)=\iint_{-\infty}^{+\infty} s(x-\xi, y-\eta) o(\xi, \eta) \mathrm{d} \xi \mathrm{d} \eta,
$$

where $i(x, y)$ is the output of the system (image) corresponding to the given input $o(x, y)$ (object).

In the Fourier space Eq. (1) becomes

$$
F\{i\}\left(\nu_{1}, \nu_{2}\right)=F\{s\}\left(\nu_{1}, \nu_{2}\right) F\{o\}\left(\nu_{1}, \nu_{2}\right),
$$

where $F\{g\}$ denotes the Fourier transform of the function $g(x$, y).

As soon as we consider circularly symmetric systems, it is advantageous to introduce polar coordinates, both in the data spaces $(r, \theta)$ and in the Fourier space $(\omega, \phi)$.

In these coordinates the Fourier transform of $g(x, y)$ is expressed by

$$
F\{g\}(\omega, \phi)=\int_{0}^{2 \pi} \int_{0}^{+\infty} g(r, \phi) \exp [i 2 \pi \omega r \cos (\theta-\phi)] r \mathrm{~d} r \mathrm{~d} \theta .
$$

Let $s(r, \theta) \equiv s(r)$ be the point-spread function of our system. From Eq. (3) its transfer function will be $2 \pi \tilde{S}(\omega)$, where $\tilde{S}(\omega)$ is the zero-order Hankel transform ${ }^{15}$ of $s(r)$,

$$
\tilde{S}(\omega)=\int_{0}^{+\infty} r s(r) J_{0}(2 \pi \omega r) \mathrm{d} r .
$$

In the following we consider a class of objects of finite support belonging to $L_{2}(R)$, where $R=\left\{(x, y): x^{2}+y^{2} \leq\right.$ $1\}$. 
Let us develop object and image into circular harmonics:

$o(r, \theta)=\sum_{N=-\infty}^{+\infty} O_{N}(r) \exp (i N \theta), \quad 0 \leqq r \leqq 1, \quad 0 \leqq \theta \leqq 2 \pi ;$

$i(r, \theta)=\sum_{N=-\infty}^{+\infty} I_{N}(r) \exp (i N \theta), \quad 0 \leqq r \leqq+\infty, \quad 0 \leqq \theta \leqq 2 \pi$.

The Fourier transforms of the above series are written as

$$
\begin{gathered}
F\{o\}(\omega, \phi)=2 \pi \sum_{N=-\infty}^{+\infty}(-i)^{N} \tilde{O}_{N}(\omega) \exp (i N \phi), \\
F\{i\}(\omega, \phi)=2 \pi \sum_{N=-\infty}^{+\infty}(-i)^{N} \tilde{I}_{N}(\omega) \exp (i N \phi), \\
\text { for } 0 \leqq \omega \leqq+\infty, \quad 0 \leqq \phi \leqq 2^{\pi},
\end{gathered}
$$

where $\tilde{O}_{N}(\omega)$ and $\tilde{I}_{N}(\omega)$ are the $n$ th-order Hankel transforms of $O_{N}(r)$ and $I_{N}(r)$,

$$
\tilde{O}_{N}(\omega)=\int_{0}^{1} r O_{N}(r) J_{N}(2 \pi \omega r) \mathrm{d} r,
$$

with inverse transform

$$
I_{N}(r)=4 \pi^{2} \int_{0}^{+\infty} \omega \tilde{I}_{N}(\omega) J_{N}(2 \pi \omega r) \mathrm{d} \omega
$$

From Eqs. (1) and (2) we immediately obtain that

$$
I_{N}(r)=8 \pi^{3} \int_{0}^{+\infty} \omega \tilde{S}(\omega) \tilde{Q}_{N}(\omega) J_{N}(2 \pi \omega r) \mathrm{d} \omega
$$

Substituting Eq. (7) into Eq. (9), we have a relationship between harmonics of the same order,

$$
I_{N}(r)=\int_{0}^{1} S_{N}(\rho, r) O_{N}(\rho) \mathrm{d} \rho \equiv\left(\hat{S}_{N} O_{N}\right)(r),
$$

$\hat{S}_{N}$ being a shift-variant integral operator.

By now the problem is reduced to the inversion of a countable set of 1-D integral operators. This problem can be approached in different ways; we adopt an iterative procedure. Through the eigenfunction analysis of the operators involved we reach a deep comprehension of the reconstruction process.

\section{THE CIRCULAR BAND-LIMITING OPERATOR: EIGENFUNCTIONS AND EIGENVALUES}

In the particular case of a circular low-pass system we have

$$
i(\bar{x})=\int_{R} s_{a}(\bar{x}-\bar{y}) o(\bar{y}) \mathrm{d} \bar{y} \equiv\left(\hat{S}_{a} o\right)(\bar{x})
$$

where $\bar{x}, \bar{y}$ denote radius vectors in the object and image planes and where

$$
s_{a}(\bar{x})=\int_{P_{a}} \exp (2 \pi i \bar{\nu} \bar{x}) \mathrm{d} \bar{\nu} .
$$

Here $P_{a}$ is the pupil defined by $P_{a} \equiv\left\{\left(\nu_{1}, \nu_{2}\right): \nu_{1}^{2}+\nu_{2}^{2} \leq\right.$ $\left.a / \pi^{2}\right\}$ so that $a$ is the space-bandwidth product of the system, that is, the product of the object support area times the accessible Fourier area $a=\pi^{2} \nu_{M}{ }^{2}\left(\nu_{M}\right.$ is the maximum transmittable frequency).
Now the kernel $S_{N}$ [see Eq. (10)] becomes

$$
S_{N, a}(\rho, r)=4 a^{2} \int_{0}^{1} \omega \rho J_{N}\left(2 \pi \nu_{M} r \omega\right) J_{N}\left(2 \pi \nu_{M} \rho \omega\right) \mathrm{d} \omega .
$$

Conventional 2-D iterative image restorations use expansions in terms of the set of eigenfunctions of $\hat{S}_{a}$, i.e., the solutions of the equation

$$
\mu_{k, l} \psi_{k, l}(\bar{x})=\left(S_{a} \psi_{k, l}\right)(\bar{x})
$$

where $\mu_{k, l}$ is a doubly numerable set ${ }^{14}$ of real and positive numbers such that $\mu_{k, l} \geq \mu_{k+1, l+j}$ with $i, j \geq 0$, while $\left\{\psi_{k, l}(\bar{x})\right\}$ are the generalized spheroidal prolate wave functions, orthogonal on $R$ and complete in $L_{2}(R) .^{16}$

If we introduce the operator $T$ that truncates to the circle $R$, the imaging relation [see Eq. (11)] leads to the expression

$$
I_{k, l}=\mu_{k, l} O_{k, l}
$$

connecting the coefficients of the expansions

$$
\begin{aligned}
o(\bar{x}) & =\sum_{k, l} O_{k, l} \psi_{k, l}(\bar{x}), \\
T i(\bar{x}) & =\sum_{k, l} I_{k, l} \psi_{k, l}(\bar{x}), \quad \bar{x} \text { in } R .
\end{aligned}
$$

Now, if we use the result ${ }^{14}$ that expanding $\psi(\bar{x}) \equiv \psi(r, \theta)$ in circular harmonics

$$
\psi(r, \theta)=\sum_{N=-\infty}^{+\infty} R_{N}(r) \exp (i N \theta),
$$

the $R_{N}(r)$ satisfy (we may only consider $N \geq \theta$ )

$$
\gamma_{N, n} R_{N, n}(r)=\int_{0}^{1} J_{N}\left(2 \pi \nu_{M} \rho r\right) R_{N, n}(\rho) \rho \mathrm{d} \rho,
$$

where $\gamma_{N, n}$ and $\mu_{N, n}$ are related by

$$
\mu_{N, n}=4 a^{2}\left|\gamma_{N, n}\right|^{2} \text {. }
$$

Comparing Eqs. (18) and (13) we immediatly see that the functions $R_{N, n}(r)$ satisfy the equation

$$
\mu_{N, n} R_{N, n}(r)=\left(\hat{S}_{N, a} R_{N, n}\right)(r) .
$$

In other words $\left\{R_{N, n}(r)\right\}$ is a complete set of eigenfunction for $S_{N, a}$.

\section{ITERATIVE RESTORATION OF HARMONIC COMPONENTS}

For each harmonic component $N$, we may expand $I_{N}(r)$, $O_{N}(r)$ into the set $\left\{R_{N, n}(r)\right\}$ complete in $L_{2}(0,1)$ :

$$
\begin{aligned}
& I_{N}(r)=\sum_{n=0}^{+\infty} i_{N, n} R_{N, n}(r), \\
& O_{N}(r)=\sum_{n=0}^{+\infty} o_{N, n} R_{N, n}(r) .
\end{aligned}
$$

Then Eq. (10) simplifies to

$$
i_{N, n}=\mu_{N, n} o_{N, n} .
$$



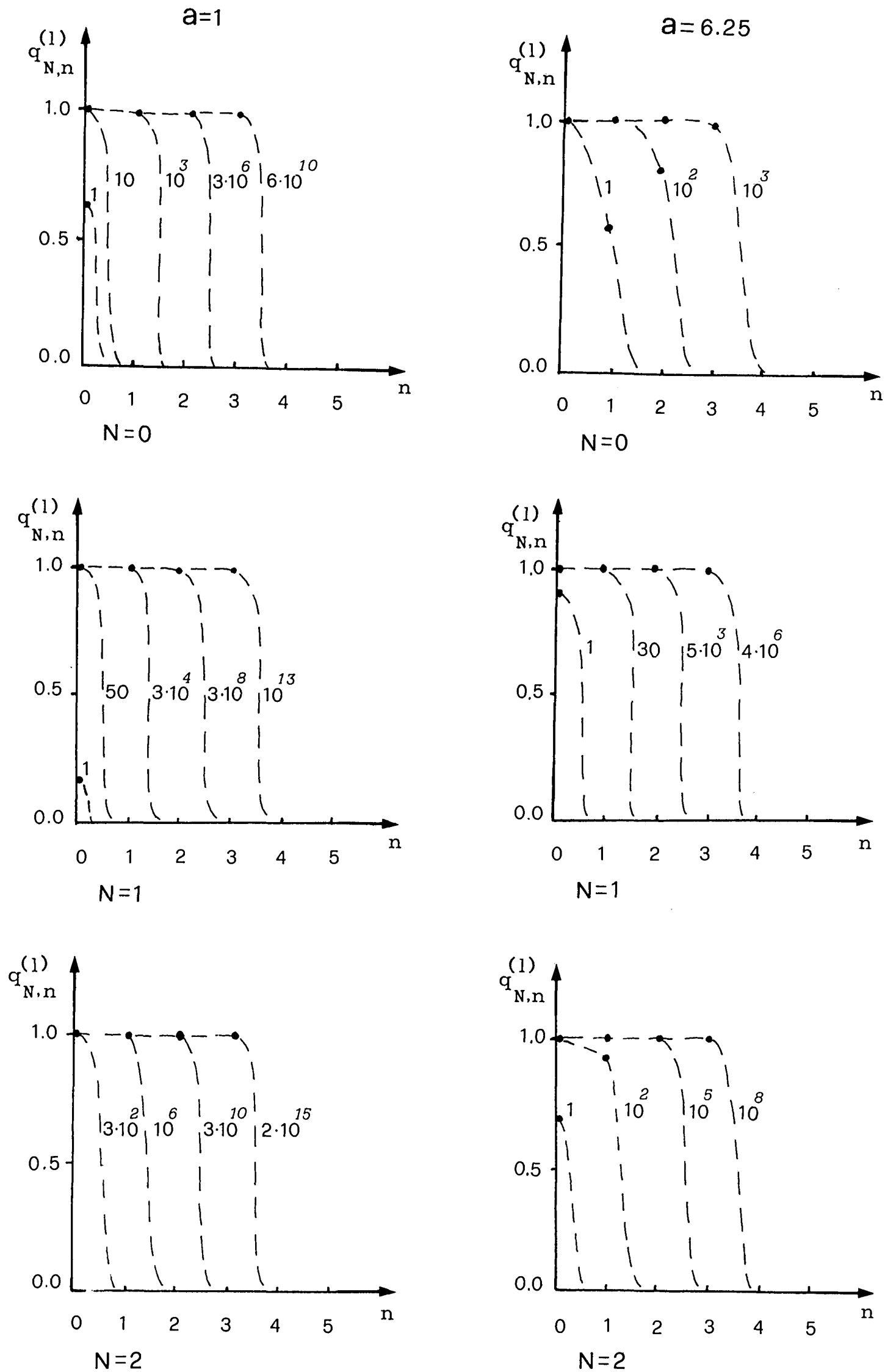

Fig. 1. Plots of $q_{N, n}{ }^{(l)}=1-\left(1-\mu_{N, n}\right)^{l}$ for different harmonics and different space-bandwidth products $a$. The number of iterations $l$ is given close to each curve [for convenience, continuous curves join the values of $q_{N, n}{ }^{(l)}$ for the same $l$ ]. 


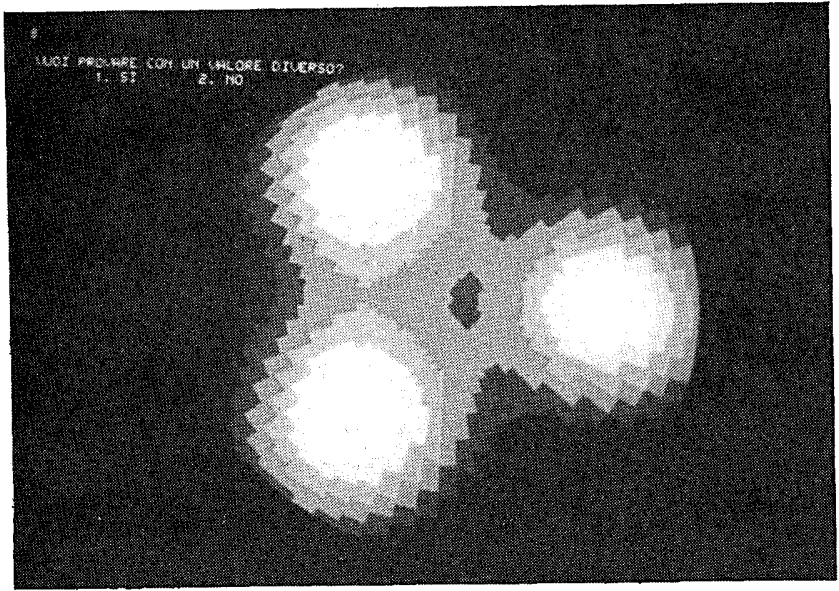

Fig. 2. Object utilized in example 1.

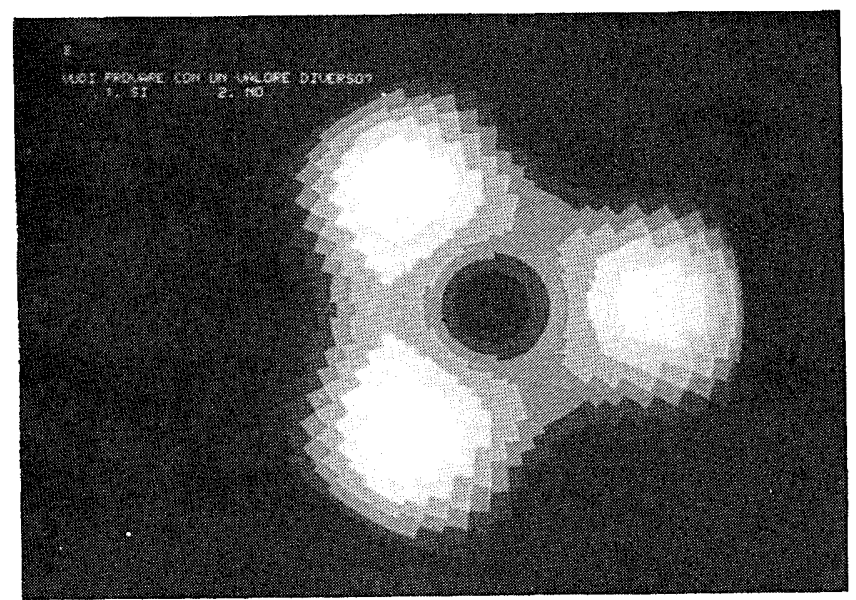

Fig. 3. Reconstruction of the image in Fig. 4 obtained by iterating the first seven harmonics according to Table 1.

Now, defining the operator $\hat{K}_{N, a}$ as

$$
\left(\hat{K}_{N, a} o\right)(x)=\int_{0}^{1}\left\{\delta(x-y)-S_{N, a}(x, y)\right\}_{o}(y) \mathrm{d} y,
$$

where $\delta(x)$ is the Dirac delta function, we may use the formal identity [see Eq. (10)]

$$
I_{N}(r)=O_{N}(r)-\left(\widehat{K}_{N, a} O_{N}\right)(r) \quad \text { for } r \text { in }[0,1]
$$

to get the iteration scheme ${ }^{6}$

$$
\begin{aligned}
O_{N}^{(l)}(r) & =I_{N}(r)+\left[\hat{K}_{N, a} O_{N}^{(l-1)}\right](r) \\
& =I_{N}(r)+\sum_{j=1}^{l-1}\left(\hat{K}_{N, a}{ }^{j} I_{N}\right)(r), \\
O_{N}{ }^{(1)}(r) & =I_{N}(r),
\end{aligned}
$$

Using Eqs. (21) and (22), we may write Eq. (25) as

$$
O_{N}{ }^{(l)}(r)=\sum_{n=0}^{+\infty} o_{N, n} q_{N, n}{ }^{(l)} R_{N, n}(r)
$$

where

$$
q_{N, n}^{(l)}=1-\left(1-\mu_{N, n}\right)^{l} .
$$

Since $0 \leq \mu_{N, n} \leq 1$, it holds that

$$
\lim _{l \rightarrow+\infty} q_{N, n}(l)=1 \quad \text { for each } N, n,
$$

which ensures the convergence of our iteration to the object's harmonic $O_{N}(r)$.

It is perhaps worth pointing out that the convergence just proved does not guarantee a meaningful result in any practical situation when the image is not perfectly known. This behavior is due to the well-known ill posedness of the mathematical problem of inverting a Fredholm integral equation of the first kind [Eq. (1)].

Theory of regularization can be used in order to restore the continuity of the inverse operator $\hat{S}_{N}{ }^{-1}$, by introducing suitable constraints on the set of acceptable solutions. ${ }^{10}$

On the other hand, in any practical reconstruction only a finite number of iterations is performed, and this regularizes the problem ${ }^{17}$ as well. Comparison of Eqs. (21) and (26) shows that $O_{N}(l)(r)$ differs from $O_{N}(r)$ in having each coefficient $o_{N, n}$ multiplied by the weighting factor $q_{N, n}{ }^{(l)}$.

In Fig. 1 we have plotted the values $q_{N, n}{ }^{(l)}$ for the first values of $N, n$ and different space-bandwidth products $a$; the iteration number $l$ is given close to each curve.

We have $q_{N, n}{ }^{(l)}=\mu_{N, n}$. As the number of iterations $l$ increases, the $q_{N, n}{ }^{(l)}$ approach one, showing a characteristic step-function behavior with respect to $n$, that is, the weighting factors are very close to unity for any index value between zero and a certain critical value, after which they drop to nearly zero values.

As one can see, for a given space-bandwidth product $a$, the critical index decreases with increasing harmonic index $N$. This signifies that, as is obvious, the higher the harmonic is, the more degraded the associated information is. If we consider the image corrupted by noise uniformly distributed over the angular harmonics, the modes that are multiplied by small

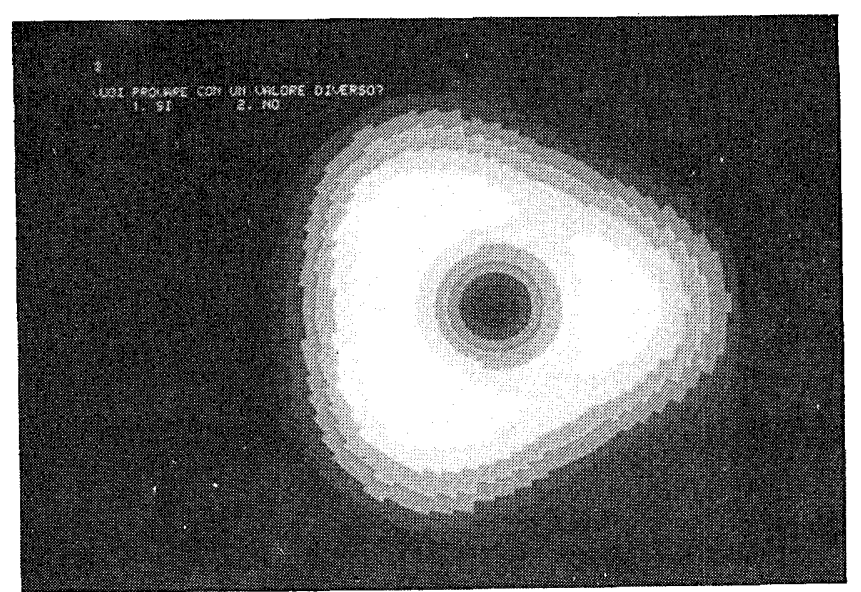

Fig. 4. Image of the object in Fig. $2(a=10)$.

Table 1. Numbers of Iterations Settled with Our Criterion for Each Harmonic of the Objects in Examples 1 and 2

\begin{tabular}{crr}
\hline $\begin{array}{c}\text { Harmonic } \\
\text { (No.) }\end{array}$ & \multicolumn{2}{c}{ Numbers of Iterations } \\
\cline { 2 - 3 } Obj. 1 & Obj. 2 \\
\hline 0 & 69 & 37 \\
1 & 291 & 169 \\
2 & 63 & 339 \\
3 & 420 & 68 \\
4 & 3498 & 289 \\
5 & 6784 & 2375 \\
6 & 796 & 1362 \\
\hline
\end{tabular}



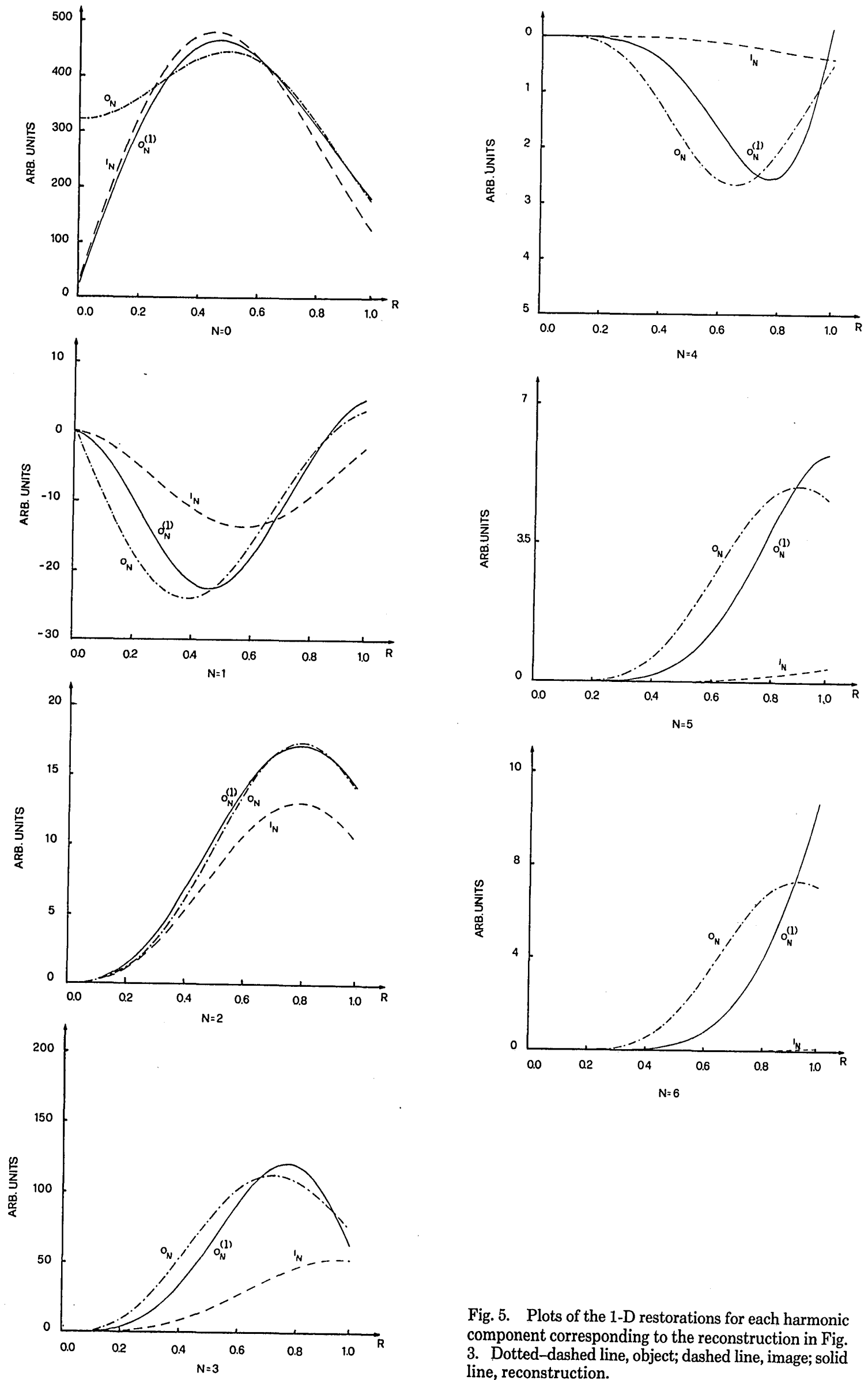

Fig. 5. Plots of the 1-D restorations for each harmonic component corresponding to the reconstruction in Fig. 3. Dotted-dashed line, object; dashed line, image; solid line, reconstruction. 


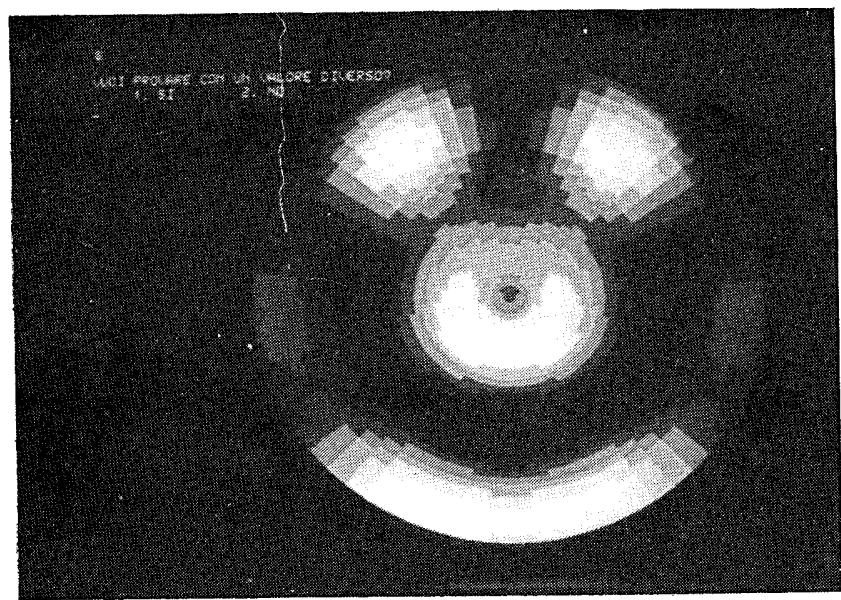

Fig. 6. Reconstruction of the image in Fig. 8 obtained by iterating the first seven harmonics according to Table 1.

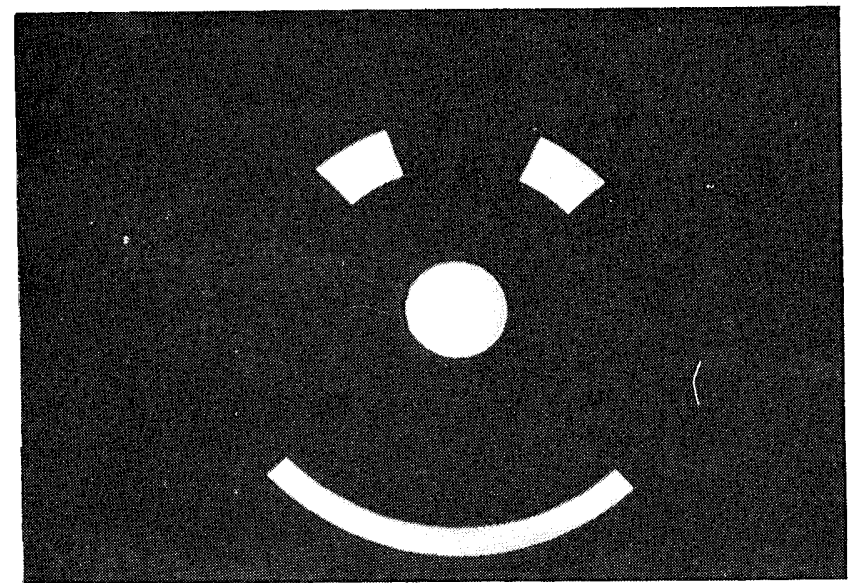

Fig. 7. Object utilized in example 2.

eigenvalues [see Eq. (22)] will not in practice be recoverable. Besides varying the harmonic $N$, we need different numbers of iterations to recover a mode with a specified index $n$. For $a=1$ about $l=10,50,300$ iterations are necessary to bring the coefficients $q_{N, 1}{ }^{(l)}$ close to one for the harmonics $N=0,1,2$, respectively (Fig. 1). Restoration of higher-order harmonics would likely mainly amplify the noise and thus should be avoided.

A different number of iterations is also required for each harmonic to obtain the same relative resolution improvement. For example, with $a=6.25$ about 200 iterations are required for the harmonic $N=0$ to double the resolution (i.e., the number of modes that are present in the image), whereas for $N=1$ we need fewer than 30 iterations.

\section{EXPERIMENTAL RESULTS}

The proposed method has been tested on some 2-D target objects. A number of noise-free images were created on a polar grid consisting of 64 angular divisions and 40 radial divisions of the domain $R$.

For each harmonic we evaluated the optimal number of iterations by means of the following criterion. Iteration of the $N$ th harmonic ends as soon as the relative improvement for iteration $e_{N}^{(l)}$, defined as

$$
\begin{aligned}
e_{N}{ }^{(l)}=\sum_{k=1}^{m}\{ & O_{N}{ }^{(l)}(k \Delta r) \\
& \left.\quad-O_{N}{ }^{(l-1)}(k \Delta r)\right\}^{2} / \sum_{k=1}^{m}\left\{O_{N}^{(1)}(k \Delta r)\right\}^{2},
\end{aligned}
$$

$m$ being the number of radial divisions, becomes smaller than a given treshold.

\section{Example 1}

The object consists of three Gaussian functions centered at different points around the origin of the plane $(x, y)$ and simulating three bright spots (Fig. 2). The minimum relative improvement for iteration is set to be $10^{-7}$.

The reconstruction (Fig. 3) of an image (Fig. 4) obtained through a system with space-bandwidth product $a=10$ is calculated by iteration of the first seven harmonics only. The higher-order harmonics of the object are in fact virtually suppressed by the system so that their contribution to the image is nearly zero. As it can be seen in Table 1, the optimal number of iterations is strongly different from one harmonic to the other. A comparison among the one-dimensional restorations of the first seven harmonics (Fig. 5) reveals how the relative resolution improvement with respect to the image harmonics $I_{N}(r)$ increases with the order $N$, as we outlined in Section 4.

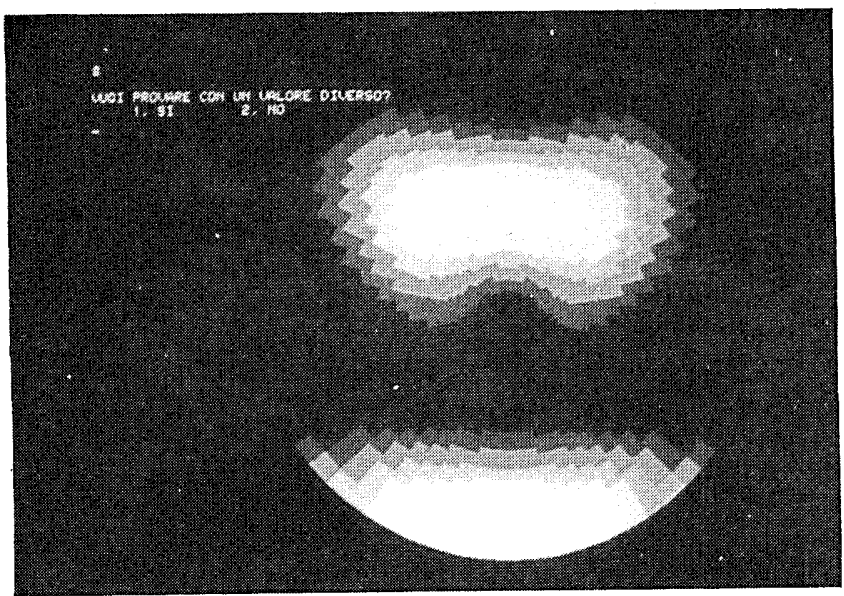

Fig. 8. Image of the object in Fig. $7(a=15)$.

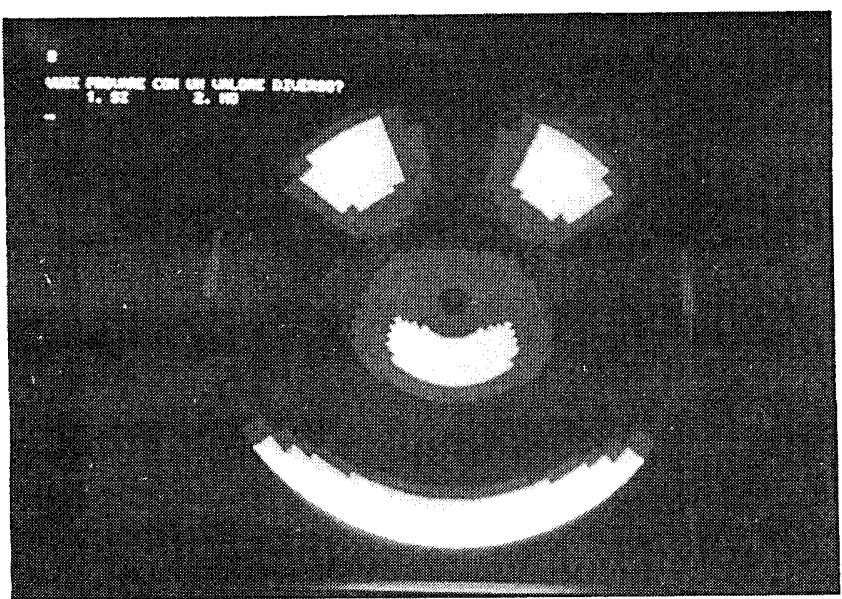

Fig. 9. Image of the object in Fig. $7(a=30)$. 


\section{Example 2}

The reconstruction (Fig. 6) of the target object shown in Fig. 7 is obtained from an image with $a=15$ (Fig. 8). Again, only the first seven harmonics turned out to be significantly present and recoverable. The threshold for the relative improvement $e_{N}{ }^{(l)}$ is now set to $10^{-6}$, and the corresponding numbers of iterations are reported in Table 1.

The same considerations as in example 1 on the numbers of iterations and resolution improvement for each harmonic are still valid.

For comparison we also report the image (Fig. 9) of the object in Fig. 7 obtained through a system with space-bandwidth product $a=30$. It can be seen that this image (Fig. 9) and the reconstruction (Fig. 6) are qualitatively similar. Therefore we conclude that the obtained superresolution has allowed for a near doubling of the effective space-bandwidth product. On the other hand, it should be clear that the increase of resolution depends on the angular spectrum of the object.

\section{CONCLUSIONS}

Using the circular symmetry of the problem, 2-D iterative superresolution is performed through separate $1-D$ reconstructions of the harmonic components of the object imaged through a circularly band-limited system.

Our method has the following important advantages:

1. It avoids interpolation errors when image data are given on a polar grid.

2. The number of required computations may also be considerably reduced. If, for instance, we need $L$ iterations to recover a particular high-frequency angular information with a conventional Gerchberg algorithm, ${ }^{11}$ the number of operations will be proportional to $L M^{2} \log _{2} M\left(M^{2}\right.$ is the number of data), since each iteration requires a $2-\mathrm{D}$ fast Fourier transform (FFT). On the other hand, if we iterate separately only the harmonic components of interest, we need a number of iterations of order $L M^{2}$ for each component, since the operator $\widehat{K}_{N, a}$ is shift variant and $L$ is allowed to vary from one harmonic to the other. For large $M$ and strongly varying $L$ the reduction of complexity is relevant.

3. Our algorithm also noticeably reduces the access time to data stored in a sequential memory, because of its lowering the dimensionality of the problem. With this method, the handling of large matrices as is required by methods based on 2-D FFT can be avoided.

4. If the image is noisy, then iteration of high harmonic components would produce only an amplification of the noise. This iteration is implicit in the 2-D algorithms and imposes a limit on the number of iterations. The limit can be considerably increased, though, in the 1-D restoration of selected harmonic components whose signal-to-noise ratio is not too low.

A minor disadvantage of the method can be seen in the increased complexity of evaluating the kernel $S_{N, a}$ [Eq. (13)] used in our iteration, in comparison with the kernel $s_{a}$ [Eq. (12)]. However, this does not significantly affect the performances of the algorithm, since the values of $S_{N, a}$ are to be computed only once for each harmonic component.
Superresolution of some computer-generated images has been performed to show the validity of the proposed method. These experimental results confirm how the computational effort is, in general, strongly different from one harmonic to the other. Consequently, one can optimize the number of iterations to be carried out for each harmonic independently.

\section{ACKNOWLEDGMENTS}

We wish to thank F. Gori, University of Rome, and D. Psaltis, California Institute of Technology, for helpful discussions. We also thank the Department of Physics of the University of Rome for making available to the authors its computing facilities.

* Present address, Department of Mathematics, Stanford University, Stanford, California 94305.

\section{REFERENCES}

1. J. L. C. Sanz and T. S. Huang, "Unified Hilbert space approach to iterative least-squares linear signal restoration," J. Opt. Soc. Am. 73, 1455-65 (1983).

2. A. M. Darling, T. J. Hall, and M. A. Fiddy, "Stable, noniterative object reconstruction from incomplete data using $a$ priori knowledge," J. Opt. Soc. Am. 73, 1466-1469 (1983).

3. L. S. Joyce and W. L. Root, "Precision bounds in superresolution processing," J. Opt. Soc. Am. A 1, 149-168 (1984).

4. M. Bertero, C. de Mol, E. R. Pike, and J. G. Walker, "Resolution in diffraction-limited imaging, a singular value analysis. IV. The case of uncertain localization or nonuniform illumination of the object," Opt. Acta, 31, 923-945 (1984).

5 . R. W. Gerchberg, "Super-resolution through error energy reduction," Opt. Acta 21, 709-720 (1974).

6. P. de Santis and F. Gori, "On an iterative method for super-resolution," Opt. Acta 22, 691-695 (1975).

7. A. Papoulis, "A new algorithm in spectral analysis and bandlimited signal extrapolation," IEEE Trans. Circuits Syst. CAS-22, $735-742(1975)$.

8. D. C. Youla, "Generalized image restoration by the method of alternating orthogonal projections," IEEE Trans. Circuits Syst. CAS-25, 694-702 (1978).

9. G. Cesini, G. Guattari, G. Lucarini, and C. Palma, "An iterative method for restoring noisy images," Opt. Acta 25, 501-508 (1978).

10. M. Bertero, C. de Mol, and G. A. Viano, "The stability of inverse problems," in Inverse Scattering Problems in Optics, Vol. 20 of Topics in Current Physics, H. P. Baltes, ed. (Springer-Verlag, Berlin, 1980), pp. 161-214.

11. R. J. Marks, II, "Gerchberg's extrapolation algorithm in two dimensions," Appl. Opt. 20, 1815-1820 (1981).

12. D. C. Youla and H. Webb, "Image restoration by the method of convex projections: part 1-Theory," IEEE Trans. Med. Imaging MI-1, 81-94 (1982).

13. M. I. Sezan and H. Stark, "Image restoration by the method of convex projections: part 2-Applications and numerical results," IEEE Trans. Med. Imaging MI-1, 95-101 (1982).

14. D. Slepian, "Prolate spheroidal wave functions, Fourier analysis and uncertainty. IV: Extensions to many dimensions; generalized prolate spheroidal wave functions," Bell Syst. Tech. J. 43, 3009-3057 (1964).

15. A. Papoulis, Systems and Transforms with Applications in Optics (McGraw-Hill, New York, 1968).

16. F. Riesz and B. S. Nagy, Functional Analysis (Ungar, New York, 1951).

17. F. Gori and G. Guattari, "Signal restoration for linear systems with weighted inputs. Singular value analysis for two cases of low-pass filtering," Inverse Problems 1, 67-85 (1985). 


\section{G. Gregori}

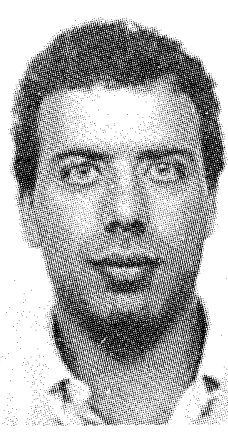

G. Gregori was born in Rome, Italy, in 1961. He received the Laurea degree in electrical engineering in 1984 from the University of Rome. After his graduation he spent a year at the Fondazione "Ugo Bordoni," Rome, on. a research grant. $\mathrm{He}$ is currently a graduate student in mathematics at Stanford University, Stanford, California. His research interests include nonlinear optics and electromagnetic inverse problems.

\section{S. Wabnitz}

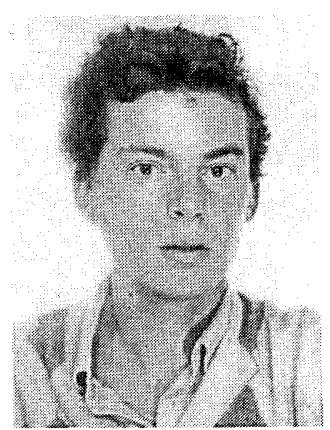

S. Wabnitz was born in Rome, Italy, in 1958. He received the Laurea degree in electrical engineering in 1982 from the University of Rome and the M.S. degree in electrical engineering in 1983 from the California Institute of Technology, Pasadena. He joined the Fondazione "Ugo Bordoni," Rome, in 1983. His research fields include electromagnetic inverse problems, propagation in optical fibers, and nonlinear optics. 